UDC 512.542:530.145

PACS 03.65.Fd, 03.65.Ud, 03.65.Aa

DOI: $10.22363 / 2658-4670-2021-29-4-347-360$

\title{
Quantum mereology in finite quantum mechanics
}

\author{
Vladimir V. Kornyak \\ Laboratory of Information Technologies \\ Joint Institute for Nuclear Research \\ 6, Joliot-Curie St., Dubna, Moscow Region, 141980, Russian Federation
}

(received: August 15, 2021; accepted: September 22, 2021)

Any Hilbert space with composite dimension can be factored into a tensor product of smaller Hilbert spaces. This allows us to decompose a quantum system into subsystems. We propose a model based on finite quantum mechanics for a constructive study of such decompositions.

Key words and phrases: quantum mereology, closed quantum system, quantum subsystems, finite quantum mechanics, quantum entanglement, energy

\section{Introduction}

Mereology is the study of the part-to-whole and part-to-part relations within a whole. In quantum mereology, the whole is a closed quantum system ("the Universe") ${ }^{1}$ in a given pure state, undergoing a given unitary (Schrödinger) evolution. Quantum mereology studies the interrelations between singled out subsystems of the Universe ("observable system", "observer", "environment", etc.), the emergence of geometry and even time (Page-Wootters mechanism [1]) from quantum entanglement, and other fundamental issues of quantum mechanics [2]-[4].

The division of the whole into parts is somewhat arbitrary and depends on the used separation criteria. There are two different facets of the separability between quantum systems.

1. Quantum systems are separated if the interaction energy between them is small. This is a more visible, material criterion that agrees well with the usual concept of locality. Quantitatively, the interaction energy between subsystems $A$ and $B$ can be represented as

$$
\Delta E(A, B)=E(A \cup B)-E(A)-E(B) .
$$

(C) Kornyak V.V., 2021

This work is licensed under a Creative Commons Attribution 4.0 International License http://creativecommons.org/licenses/by/4.0/

\footnotetext{
${ }^{1}$ Obviously, in the exact sense, closed systems do not exist (or they are fundamentally unobservable), with the possible exception of the Universe as a whole.
} 
2. Quantum systems are separated if the quantum correlations between them are small. This criterion is more subtle and has non-local manifestations. Quantitatively, the entanglement between subsystems can be described, for example, by mutual information

$$
\mathcal{J}(A, B)=S(A)+S(B)-S(A \cup B),
$$

where $S$ denotes entropy.

There is a certain structural similarity between expressions (1) and (2). However, they describe completely different types of connections between subsystems.

For example, in the Page-Wootters model of emergent time, it is assumed that the whole timeless Universe is divided into two subsystems: the "clock", $C$, and the rest of the Universe, $R$. It is assumed that the Hamiltonian of the Universe has the form $H=H_{C} \otimes \mathbb{1}_{R}+\mathbb{1}_{C} \otimes H_{R}$, which means that the interaction energy between $C$ and $R$ is zero. On the other hand, the existence of nontrivial quantum correlations between $C$ and $R$ is assumed.

It would be interesting to take a closer look at the interplay between these two different, energy and information, aspects of quantum separability.

We develop and implement algorithms based on computer algebra techniques to perform the following. An isolated quantum system, constructed in the framework of finite quantum mechanics, is decomposed into a tensor product of subsystems. By reducing the "universe" quantum state, we obtain mixed states for subsystems. This allows us to study energy interactions and quantum correlations between subsystems and their time evolution.

\section{Decomposition of a quantum system}

Tensor product of Hilbert spaces. The (global) Hilbert space $\mathcal{H}$ of a $K$ component quantum system is the tensor product of the (local) Hilbert spaces $\mathcal{H}_{k}$ of the components:

$$
\mathcal{H}=\bigotimes_{k=1}^{K} \mathcal{H}_{k}
$$

If $\operatorname{dim} \mathcal{H}=\mathcal{N}$ and $\operatorname{dim} \mathcal{H}_{k}=d_{k}$, then $\mathcal{N}=\prod_{k=1}^{K} d_{k}$.

For any $d$-dimensional Hilbert space, the $i$ th orthonormal basis element is denoted by $|i\rangle$, that is,

$$
|0\rangle=(1,0, \ldots)^{\top},|1\rangle=(0,1,0, \ldots)^{\top}, \ldots,|d-1\rangle=(0,0, \ldots, 1)^{\top} .
$$

Tensor monomials of local basis elements form an orthonormal basis in the global Hilbert space:

$$
|i\rangle=\left|i_{1}\right\rangle \otimes \cdots \otimes\left|i_{k}\right\rangle \otimes \cdots \otimes\left|i_{K}\right\rangle,
$$


where $|i\rangle \in \mathcal{H},\left|i_{k}\right\rangle \in \mathcal{H}_{k}$ and

$$
i=i_{1} \prod_{m=2}^{K} d_{m}+\ldots+i_{k} \prod_{m=k+1}^{K} d_{m}+\ldots+i_{K} .
$$

Tensor factorization of a Hilbert space. We can reverse the procedure, since (4) is a one-to-one correspondence: the sequence $i_{1}, \ldots, i_{K}$ is uniquely recovered from $i$ by a simple procedure based on formula (5):

$$
\begin{aligned}
& k \leftarrow K, \tilde{\imath} \leftarrow i \\
& \text { while } k \geqslant 1 \text { do } \\
& \quad i_{k} \leftarrow \tilde{\imath} \bmod d_{k}, \tilde{\imath} \leftarrow\left\lfloor\tilde{\imath} / d_{k}\right\rfloor, k \leftarrow k-1 \\
& \text { end while }
\end{aligned}
$$

Given an orthonormal basis in an $\mathcal{N}$-dimensional Hilbert space $\mathcal{H}$ and a decomposition $\mathcal{N}=d_{1} \cdots d_{K}$, we can construct a particular bijection of the form (3).

When constructing a bijection, we must take into account the freedom in the choice of bases in Hilbert spaces. Any two orthonormal bases are related by a unitary transformation.

It is easy to show that general unitary changes of bases in all involved spaces are equivalent to a single change in the global space.

Namely, any vector of the global space can be represented as a sum of tensor products of elements of local spaces

$$
|\psi\rangle=\sum_{\ell} \bigotimes_{k=1}^{K}\left|\psi_{k}^{\ell}\right\rangle,\left|\psi_{k}^{\ell}\right\rangle \in \mathcal{H}_{k},|\psi\rangle \in \mathcal{H} .
$$

Applying unitary transformations to all vectors in (7) and using the properties of the tensor product, we have

$$
\begin{gathered}
U|\psi\rangle=\sum_{\ell} \bigotimes_{k=1}^{K} U_{k}\left|\psi_{k}^{\ell}\right\rangle=\bigotimes_{k=1}^{K} U_{k} \sum_{\ell} \bigotimes_{k=1}^{K}\left|\psi_{k}^{\ell}\right\rangle \\
\Downarrow \\
U^{\prime}|\psi\rangle=\sum_{\ell} \bigotimes_{k=1}^{K}\left|\psi_{k}^{\ell}\right\rangle, \text { where } U^{\prime}=\left(\bigotimes_{k=1}^{K} U_{k}\right)^{-1} U .
\end{gathered}
$$

Thus, to specify the factorization of the Hilbert space $\mathcal{H}$ we need two things ${ }^{2}$

1. an integer decomposition $\operatorname{dim} \mathcal{H}=d_{1} \cdots d_{K}$, and

2. a unitary transformation $U$, which fixes a basis in $\mathcal{H}$.

\footnotetext{
${ }^{2}$ Another approach, in which the tensor factorization of a Hilbert space is specified by a set of observables, was proposed in [5], [6].
} 
Decomposition of a pure quantum state. Any mixed state of a quantum system can be obtained from a pure state in a larger Hilbert space by taking a partial trace. It is natural to assume that at the fundamental level the state of an isolated system must be pure. ${ }^{3}$ For a given factorization $\mathcal{H}=\mathcal{H}_{1} \otimes \cdots \otimes \mathcal{H}_{K}$, we introduce the set of indices (which can be thought of as "geometric points")

$$
X=\{1, \ldots, K\} .
$$

Subsystems are identified with subsets $A \subseteq X$. The density matrix of the pure state $|\psi\rangle \in \mathcal{H}$ of the entire system is $\rho_{X}=\frac{|\psi\rangle\langle\psi|}{\langle\psi \mid \psi\rangle}$. According to the laws of quantum mechanics, the statistical behavior of the subsystem $A$ is correctly described by the reduced density matrix $\rho_{A}=\operatorname{tr}_{X \backslash A} \rho_{X}$ calculated by taking the partial trace over the complement to $A$.

In more detail, the calculation of the reduced density matrix is as follows. According to (4), the basis of the global Hilbert space can be represented as the Cartesian product of the local bases

$$
B_{X}=\prod_{k \in X} B_{k} .
$$

In a similar way we introduce the sets

$$
B_{A}=\prod_{k \in A} B_{k} \text { and } B_{X \backslash A}=\prod_{k \in X \backslash A} B_{k} .
$$

In components, the global density matrix can be written as

$$
\rho_{X}=\left(\rho_{X}\right)_{i_{X} j_{X}}\left|i_{X}\right\rangle\left\langle j_{X}\right|
$$

where $i_{X} \simeq\left\{i_{1}, \ldots, i_{K}\right\} \in B_{X}$ and $j_{X} \simeq\left\{j_{1}, \ldots, j_{K}\right\} \in B_{X}$, and the equivalence $\simeq$ is provided by formula (5) and procedure (6). The procedure for calculating the reduced density matrix is obvious from the formula

$$
\left(\rho_{A}\right)_{i_{A} j_{A}}=\sum_{\substack{m_{X \backslash A} \in B_{X \backslash A} \\ i_{X}=i_{A} \sqcup m_{X \backslash A} \\ j_{X}=j_{A} \sqcup m_{X \backslash A}}}\left(\rho_{X}\right)_{i_{X} j_{X}} \text {, where } i_{A}, j_{A} \in B_{A} .
$$

\section{Finite quantum mechanics}

We use a version of quantum theory [8]-[10] in which the groups of unitary evolutions are replaced by linear representations of finite groups, and the field

\footnotetext{
${ }^{3}$ This belief (an instance of Occam's razor), expressed by the metaphor "Church of the Larger Hilbert Space" (J.A. Smolin), allows one to obtain all probabilities in quantum theory from the only fundamental probability that is described by Gleason's theorem [7] (Born's rule).
} 
of complex numbers is replaced by its dense constructive subfields, which naturally arise from the non-negative integers and roots of unity.

Permutation Hilbert space. Any linear (hence unitary) representation of a finite group is a subrepresentation of some permutation representation. This implies that the formalism of quantum mechanics can be completely ${ }^{4}$ reproduced based on permutations of some set

$$
\Omega=\left\{e_{1}, \ldots, e_{\mathcal{N}}\right\} \cong\{1, \ldots, \mathcal{N}\}
$$

of primary ("ontic") objects on which a permutation group $G \leqslant \mathrm{~S}_{\mathcal{N}}$ acts.

The Hilbert space on $\Omega$, needed for calculations in quantum theory, can be most economically constructed on the basis of two primitive concepts:

1. natural numbers $\mathbb{N}=\{0,1, \ldots\}$, abstraction of counting, and

2. roots of unity, abstraction of periodicity.

To construct a field $\mathcal{F}$ sufficient for all the needs of the quantum formalism, in particular, for splitting any representation of any subgroup of $G$ into irreducible components, we can proceed as follows. We extend the semiring $\mathbb{N}$ to the ring $\mathbb{N}\left[\zeta_{\ell}\right]$, where $\zeta_{\ell}$ is the $\ell$ th primitive root of unity, and $\ell$ is the LCM of the periods of the elements of $G$. The algebraic integer $\zeta_{\ell}$ can be written in complex form as $\zeta_{\ell}=\mathrm{e}^{2 \pi \mathrm{i} / \ell}$. Finally, constructing the quotient field of the ring $\mathbb{N}\left[\zeta_{\ell}\right]$, we arrive at the cyclotomic extension of the rationals $\mathcal{F}=\mathbb{Q}\left(\mathrm{e}^{2 \pi \mathrm{i} / \ell}\right)$. For $\ell>2$, the field $\mathcal{F}$, being a dense subfield of $\mathbb{C}$, is empirically indistinguishable from $\mathbb{C}$.

Treating the set $\Omega$ as a basis, we obtain an $\mathcal{N}$-dimensional Hilbert space $\mathcal{H}_{\mathcal{N}}$ over $\mathcal{F}$. The action of $G$ on $\Omega$ determines the permutation representation $\mathcal{P}$ in $\mathcal{H}_{\mathcal{N}}$ by the matrices $\mathcal{P}(g)_{i, j}=\delta_{i g, j}$, where $i g$ denotes the (right) action of $g \in G$ on $i \in \Omega$.

Decomposition of permutation representation. The permutation representation of any group $G$ has the trivial one-dimensional subrepresentation in the space spanned by the all-ones vector

$$
|\omega\rangle=(\underbrace{1,1, \ldots, 1}_{\mathcal{N}})^{\top} .
$$

The complement to the trivial subrepresentation is called the standard representation. The operator of projection onto the $(\mathcal{N}-1)$-dimensional standard space $\mathcal{H}_{\star}$ has the form

$$
P_{\star}=\mathbb{1}_{\mathcal{N}}-\frac{|\omega\rangle\langle\omega|}{\mathcal{N}} .
$$

Quantum mechanical behavior (interference, etc.) manifests itself precisely in $\mathcal{H}_{\star}$. Tom Banks made a profound observation [11] that the projection of classical permutation evolutions in the whole $\mathcal{H}_{\mathcal{N}}$ leads to truly quantum evolutions in the subspace $\mathcal{H}_{\star}$. Banks also showed that the choice $G=\mathrm{S}_{\mathcal{N}}$,

\footnotetext{
${ }^{4}$ Modulo empirically insignificant elements of traditional formalism such as infinities of various kinds.
} 
where $\mathcal{N}$ is the number of fundamental (Planck) elements, ${ }^{5}$ "can accurately reproduce all of the results of conventional quantum mechanics". In order to clarify a correspondence between finite quantum mechanics and traditional theory based on continuous unitary groups, Banks pointed out the connection between the symmetric group on $\mathcal{N}$ elements and the unitary group in $\mathcal{N}-1$ dimensions. Namely, for sufficiently large $\mathcal{N}$ (according to [12] $\mathcal{N} \geqslant 72$ ), the most general finite subgroup, $G$, of $\mathrm{SU}(\mathcal{N}-1)$ has the structure of a semidirect product of a finite Abelian group, $A$, and the group $\mathrm{S}_{\mathcal{N}}$

$$
G=A \rtimes \mathrm{S}_{\mathcal{N}}<\mathrm{SU}(\mathcal{N}-1) .
$$

Ontic vectors. $\mathrm{S}_{\mathcal{N}}$ is a rational-representation group, i.e., its every irreducible representation (the standard representation is one of them) is realizable over $\mathbb{Q}$. This means that to describe evolutions in $\mathcal{H}_{\star}$, it is sufficient to consider only vectors with rational components. ${ }^{6}$

It is easy to show that any quantum state in $\mathcal{H}_{\star}$ can be obtained as the projection of an integer vector from the non-negative orthant $\mathcal{H}_{\mathcal{N}}^{+} \subset \mathcal{H}_{\mathcal{N}}$. Let $|x\rangle=\left(x_{1}, \ldots, x_{\mathcal{N}}\right)^{\top} \in \mathcal{H}_{\mathcal{N}}^{+}$be a vector with non-negative rational components. Then its projection to $\mathcal{H}_{\star}$ is an $(\mathcal{N}-1)$-dimensional vector of the form

$$
|y\rangle=\left(y_{1}, \ldots, y_{\mathcal{N}-1}\right)^{\top}=\mathrm{P}_{\star}|x\rangle .
$$

The set $\{|0\rangle-|\mathcal{N}-1\rangle, \ldots,|\mathcal{N}-2\rangle-|\mathcal{N}-1\rangle\}$ is one of the bases in $\mathcal{H}_{\star}$, where $\{|0\rangle, \ldots,|\mathcal{N}-1\rangle\}$ is a basis in $\mathcal{H}_{\mathcal{N}}$. In this basis, equation (9) is equivalent to the set of relations

$$
y_{1}=x_{1}-x_{\mathcal{N}}, \ldots, y_{i}=x_{i}-x_{\mathcal{N}}, \ldots, y_{\mathcal{N}-1}=x_{\mathcal{N}-1}-x_{\mathcal{N}} .
$$

Obviously, any set of values $y_{1}, \ldots, y_{\mathcal{N}-1}$ can be obtained using only nonnegative values $x_{1}, \ldots, x_{\mathcal{N}}$.

Since quantum states are rays in Hilbert space, we can replace non-negative rational vectors $|x\rangle$ with natural vectors $|n\rangle=\left(n_{1}, \ldots, n_{\mathcal{N}}\right)^{\top} \in \mathbb{N}^{\mathcal{N}} \subset \mathcal{H}_{\mathcal{N}}^{+}$. To build constructive models (to remain in the finite realm), one needs to select a finite subset in $\mathbb{N}^{\mathcal{N}}$. The simplest choice is vectors with coordinates from the set $\{0,1\}$, i.e., bit strings of length $\mathcal{N}$. We call them ontic vectors or ontic states. These states are attractive for both ontological and computational reasons.

Interpreting ontic state $|q\rangle$ as a characteristic function, we can identify it with the subset $q \subset \Omega$ or, equivalently, with the partition of the ontic set (8) into two nontrivial subsets $\Omega=q \sqcup \sim q, \sim q=\Omega \backslash q$, where $\sim$ denotes

\footnotetext{
${ }^{5}$ By the current cosmological data, the number $\mathcal{N}$ is estimated as $\sim \operatorname{Exp}(\operatorname{Exp}(20))$ and $\sim \operatorname{Exp}(\operatorname{Exp}(123))$ for $1 \mathrm{~cm}^{3}$ of matter and for the entire Universe, respectively.

${ }^{6}$ Complex numbers - i.e., nontrivial elements of cyclotomic extensions - may be needed only in problems that require splitting representations of some proper subgroups of $S_{\mathcal{N}}$ into irreducible components.
} 
set complement operation (or bitwise inversion). The complete set of ontic states is $Q=2^{\Omega} \backslash\{\emptyset, \Omega\}$. The number of ontic states, $|Q|=2^{\mathcal{N}}-2$, depends exponentially on $\mathcal{N}$, so they present a fairly large set of quantum states in the standard space for large $\mathcal{N}$.

The complement operation applied to an ontic state induces a change in the sign of the corresponding quantum state in the standard space:

$$
|\psi\rangle=\mathrm{P}_{\star}|q\rangle \Longrightarrow-|\psi\rangle=\mathrm{P}_{\star}|\sim q\rangle .
$$

The inner product of normalized projections of the ontic vectors $|q\rangle$ and $|r\rangle$ onto $\mathcal{H}_{\star}$ is

$$
S(q, r) \equiv \frac{\left\langle q\left|\mathrm{P}_{\star}\right| r|q| \mathrm{P}_{\star} \mid r\right\rangle}{\sqrt{\left\langle q\left|\mathrm{P}_{\star}\right| q|q| \mathrm{P}_{\star} \mid q\right\rangle\left\langle r\left|\mathrm{P}_{\star}\right| r|r| \mathrm{P}_{\star} \mid r\right\rangle}}=\frac{\mathcal{N}\langle q \& r\rangle-\langle q\rangle\langle r\rangle}{\sqrt{\langle q\rangle\langle\sim q\rangle\langle r\rangle\langle\sim r\rangle}},
$$

where $\&$ is the bitwise AND for bit strings, and $\langle\cdot\rangle$ denotes population number (or Hamming weight). The obvious identities $\langle\sim a\rangle=\mathcal{N}-\langle a\rangle$ and $\langle a \& b\rangle+\langle a \& \sim b\rangle=\langle a\rangle$ imply the folowing symmetries with respect to the complement operations on the ontic states

$$
S(q, r)=-S(\sim q, r)=-S(q, \sim r)=S(\sim q, \sim r) .
$$

\section{Ontic and energy bases}

Ontic basis. The original permutation basis in the space $\mathcal{H}_{\mathcal{N}}$, i.e., the set $\Omega$, will be called the ontic basis. In this basis, the density matrix in $\mathcal{H}_{\star}$ associated with the ontic state $|q\rangle \in \mathcal{H}_{\mathcal{N}}$ has the form

$$
\rho_{q}^{o}=\frac{\mathrm{P}_{\star}|q\rangle\langle q| \mathrm{P}_{\star}}{\left\langle q\left|\mathrm{P}_{\star}\right| q|q| \mathrm{P}_{\star} \mid q\right\rangle}=\frac{1}{\mathcal{N}} \frac{(|q\rangle-\alpha|\omega\rangle)(\langle q|-\alpha\langle\omega|)}{\alpha(1-\alpha)},
$$

where $\alpha=\langle q\rangle / \mathcal{N}$ is the population density. There is an obvious duality: the expression for the density matrix $\rho_{\sim q}^{o}$ is obtained from (10) by replacements $q \rightarrow \sim q$ and $\alpha \rightarrow 1-\alpha$

$$
\rho_{q}^{o} \stackrel{q \rightarrow \sim \sim q}{\stackrel{\alpha \rightarrow 1-\alpha}{\longrightarrow}} \rho_{\sim q}^{o}
$$

Energy basis. In continuous quantum mechanics, the evolution of an isolated system is described by the one-parameter unitary group $U_{t}=\mathrm{e}^{-\mathbf{i} H t}$ generated by the Hamiltonian $H$ whose eigenvalues are called energy eigenvalues.

In finite quantum mechanics, the evolution is described by a cyclic group $U(g)^{t}$ generated by an element $U(g) \in \mathcal{P}(G)$, where $t$ is an integer parameter. We call the energy basis an orthonormal basis in which the matrix $U(g)$ is diagonal. 
Planck's formula $E=h \nu$ relates the energy $E$ to the frequency $\nu$, which is defined as the inverse of the period of the corresponding cyclic process.

Any permutation can be represented as a product of disjoint cycles. It is instructive to see how often cycles of different lengths occur in the group of all permutations $S_{\mathcal{N}}$. A simple combinatorial calculation shows that the total number of cycles of length $\ell$ in the whole group $S_{\mathcal{N}}$ is $\mathcal{N} ! / \ell$, and, therefore, the expected number of $\ell$-cycles in a single permutation is $1 / \ell$. That is, high-energy evolutions prevail in our permutation-based model of the Universe. ${ }^{7}$

The $\ell$-cycle matrix has the form

$$
C_{\ell}=\left(\begin{array}{ccccc}
0 & 1 & 0 & \cdots & 0 \\
0 & 0 & 1 & \cdots & 0 \\
\vdots & \vdots & \vdots & \vdots & \vdots \\
1 & 0 & 0 & \cdots & 0
\end{array}\right),
$$

i.e., $\left(C_{\ell}\right)_{i j}=\delta_{i-j+1(\bmod \ell)}$. The diagonal form of this matrix is

$$
F_{\ell} C_{\ell} F_{\ell}^{-1}=\left(\begin{array}{ccccc}
1 & 0 & 0 & \cdots & 0 \\
0 & \zeta_{\ell} & 0 & \cdots & 0 \\
0 & 0 & \zeta_{\ell}^{2} & \cdots & 0 \\
\vdots & \vdots & \vdots & \vdots & \vdots \\
0 & 0 & 0 & \cdots & \zeta_{\ell}^{\ell-1}
\end{array}\right)
$$

where $\zeta_{\ell}=\mathrm{e}^{2 \pi \mathrm{i} / \ell}$ is the $\ell$ th ("ground") primitive root of unity, and

$$
F_{\ell}=\frac{1}{\sqrt{\ell}}\left(\begin{array}{ccccc}
1 & 1 & 1 & \cdots & 1 \\
1 & \zeta_{\ell}^{-1} & \zeta_{\ell}^{-2} & \cdots & \zeta_{\ell}^{-(\ell-1)} \\
1 & \zeta_{\ell}^{-2} & \zeta_{\ell}^{-4} & \cdots & \zeta_{\ell}^{-2(\ell-1)} \\
\vdots & \vdots & \vdots & \cdots & \vdots \\
1 & \zeta_{\ell}^{-(\ell-1)} & \zeta_{\ell}^{-2(\ell-1)} & \cdots & \zeta_{\ell}^{-(\ell-1)(\ell-1)}
\end{array}\right)
$$

is the Fourier transform matrix. $F_{\ell}$ is both unitary and symmetric, therefore

$$
F_{\ell}^{-1}=F_{\ell}^{\dagger}=F_{\ell}^{*} \Longrightarrow\left(F_{\ell}^{-1}\right)_{i j}=\frac{1}{\sqrt{\ell}} \zeta_{\ell}^{(i-1)(j-1)} .
$$

In general, the matrix of the permutation representation of an element $g \in \mathrm{S}_{\mathcal{N}}$ is the direct sum of cyclic matrices $U(g)=\bigoplus_{m=1}^{M} C_{\ell_{m}}$, and the corresponding

${ }^{7}$ Of course, it would be more adequate to calculate the energy distribution for a given individual permutation evolution, but this is a more difficult combinatorial problem. 
diagonalizing matrix is $F=\bigoplus_{m=1}^{M} F_{\ell_{m}}$, which is the transition matrix from the ontic basis to the energy basis.

The density matrix of the whole system in the energy basis can be calculated from (10) by the formula $\rho_{q}^{\varepsilon}=F \rho_{q}^{o} F^{*}$.

\section{Entanglement measures}

Quantitatively, quantum correlations are described by measures of entanglement, which are based on the concept of entropy. The most commonly used in physics is the von Neumann entropy

$$
S_{1}(\rho)=-\operatorname{tr}(\rho \log \rho) .
$$

Also often used are entropies from the Rényi family [13]

$$
S_{\alpha}(\rho)=\frac{1}{1-\alpha} \log \operatorname{tr}\left(\rho^{\alpha}\right), \quad \alpha \geqslant 0, \alpha \neq 1 .
$$

The common feature of the von Neumann and Rényi entropies is their additivity on combinations of independent probability distributions determined by the eigenvalues of the density matrices. The von Neumann entropy is preferred because it additionally satisfies a stronger requirement, the chain rule for conditional entropies. In fact, the von Neumann entropy (11) can also be included in family (12) by going to the limit $\alpha \rightarrow 1$.

In our calculations, we use the 2nd Rényi entropy (also called the collision entropy) $S_{2}(\rho)=-\log \operatorname{tr}\left(\rho^{2}\right)$ for the following reasons:

- It is easy to calculate. For $n \times n$ density matrix $\rho$ we have

$$
S_{2}(\rho)=-\log \left(\sum_{i=1}^{n} \rho_{i i}^{2}+2 \sum_{i=1}^{n-1} \sum_{j=i+1}^{n}\left|\rho_{i j}\right|^{2}\right) .
$$

- $\operatorname{tr}\left(\rho^{2}\right)$ is the so-called purity of the state $\rho$.

- $\operatorname{tr}\left(\rho^{2}\right)$ coincides with the Born probability: "the system observes itself."

- $\operatorname{tr}\left(\rho^{2}\right) \equiv\langle\rho\rangle_{\rho}$ is the expectation value of the observable $\rho$ in the state $\rho$.

- $\operatorname{tr}\left(\rho^{2}\right) \equiv\|\rho\|_{\mathrm{F}}^{2}$ is the square of the Frobenius (Hilbert-Schmidt) norm of the density matrix.

The Frobenius inner product for two matrices (or Hilbert-Schmidt inner product for two operators) $a$ and $b$ is defined as $\langle a \mid b\rangle_{\mathrm{F}}=\operatorname{tr}\left(a^{\dagger} b\right)$. The corresponding Frobenius norm is $\|a\|_{\mathrm{F}}=\sqrt{\langle a \mid a\rangle_{\mathrm{F}}}$. It can be shown that any constructions used in the study of quantum correlations and based on the von Neumann entropy can be reformulated in terms of matrix metrics. 
For example, in emergent geometry models [3], [14], [15], the distances between subsystems $A$ and $B$ are described by functions of mutual information

$$
\mathcal{J}(A, B)=S_{1}\left(\rho_{A}\right)+S_{1}\left(\rho_{B}\right)-S_{1}\left(\rho_{A \cup B}\right) .
$$

Replacing in (13) the von Neumann entropy with the 2nd Rényi entropy, we obtain the expression

$$
\mathcal{J}_{2}(A, B)=S_{2}\left(\rho_{A}\right)+S_{2}\left(\rho_{B}\right)-S_{2}\left(\rho_{A \cup B}\right)
$$

whose exponential has the form

$$
\frac{\operatorname{tr}\left(\rho_{A \cup B}^{2}\right)}{\operatorname{tr}\left(\rho_{A}^{2}\right) \operatorname{tr}\left(\rho_{B}^{2}\right)} \equiv \frac{\left\|\rho_{A \cup B}\right\|_{\mathrm{F}}^{2}}{\left\|\rho_{A} \otimes \rho_{B}\right\|_{\mathrm{F}}^{2}} .
$$

Obviously, both (14) and (15), although (14) does not have a probabilistic interpretation of (13), also describe quite well the deviation from separability caused by entanglement.

\section{Some computational observations}

We are developing a $\mathrm{C}$ program for constructing tensor decompositions and calculating quantum correlations in the ontic and energy bases.

To illustrate the calculations, consider a homogeneous quantum system, i.e., a system whose Hilbert space is decomposed into a product of local spaces of the same dimension

$$
\mathcal{H}=\bigotimes_{x \in X} \mathcal{H}_{x}
$$

where $X=\{1, \ldots,|X|\}$ (a set of "geometric points"), $\operatorname{dim} \mathcal{H}_{x}=d$ for all $x \in X$.

Preliminary calculations indicate that decompositions with smaller local dimension $d$ exhibit more interesting behavior. Consider, e.g., the case $d=2$ and $|X|=23$, in which $\operatorname{dim} \mathcal{H}=8388608$. We will treat here the analogue of mutual information (14) as a measure of the distance between points. Calculations of (14) on all edges $(x, y) \in X \times X$ of the complete graph on $X$ show a spread of values by two orders of magnitude: a typical example is

$$
\mathcal{J}_{2}(x, y) \in\left[5.3 \times 10^{-8}, 7 \times 10^{-6}\right] .
$$

A large scatter in the distances between points can be considered a sign of the non-triviality of the geometry.

For the case with a slightly larger local dimension, $d=7$ and $|X|=7$ (hence $\operatorname{dim} \mathcal{H}=823543)$, similar calculations give $\mathcal{J}_{2}(x, y) \in[0.0037,0.0041]$. That is, the geometry is close to trivial - all points are almost equidistant. More detailed calculations show that the main contribution to the non-triviality of geometry is made by the local dimension $d$, and not by the number of points $|X|$.

Figure 1 shows examples of calculating the entropies of subsystems of all possible sizes for quantum systems with $d=2$, and $|X|=6$ ("small" case) and 
$|X|=12$ ("large" case). In both cases, 10 randomly generated ontic states were used - the legends show their Hamming weights. The data presented in the figure demonstrates the following features:

- The trend towards universality (weak dependence on the quantum state) with the growth of $|X|$ : visually, all graphs are almost identical in the "large" case.

- Symmetry $S_{2}\left(\rho_{A}\right)=S_{2}\left(\rho_{X \backslash A}\right)$ is a manifestation of the Schmidt decomposition [16] of a pure state: both matrices $\rho_{A}$ and $\rho_{X \backslash A}$ have identical sets of nonzero eigenvalues.

- For the subsystem size $|A|$ noticeably smaller than $|X| / 2$, the reduced state is close to the maximally mixed state: $S_{2}\left(\rho_{A}\right) \approx|A| \log d$. Recall that a maximally mixed state is a state whose density matrix describes a uniform probability distribution, i.e., all its eigenvalues are equal.
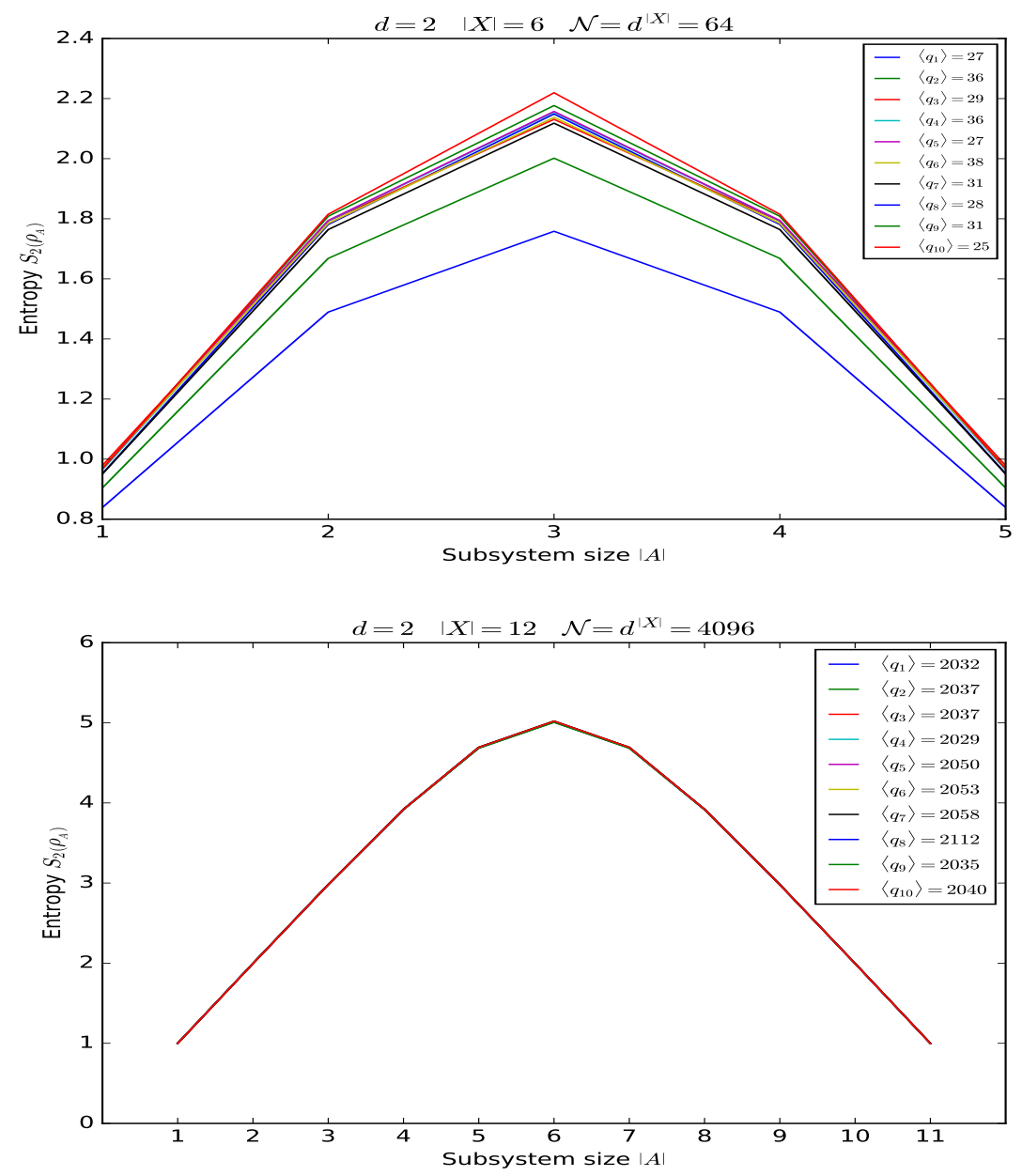

Figure 1. Entropies of subsystems 


\section{References}

[1] D. N. Page and W. K. Wootters, "Evolution without evolution: dynamics described by stationary observables," Phys. Rev. D, vol. 27, pp. 28852892, 12 Jun. 1983. DOI: 10.1103/PhysRevD.27. 2885.

[2] S. M. Carroll and A. Singh, "Quantum mereology: factorizing Hilbert space into subsystems with quasiclassical dynamics," Physical Review A, vol. 103, no. 2, Feb. 2021. DOI: 10.1103/physreva.103.022213.

[3] C. Cao, S. M. Carroll, and S. Michalakis, "Space from Hilbert space: recovering geometry from bulk entanglement," Physical Review D, vol. 95, no. 2, Jan. 2017. DOI: 10.1103/physrevd.95.024031.

[4] M. Woods, "The Page-Wootters mechanism 36 years on: a consistent formulation which accounts for interacting systems," Quantum Views, vol. 3, p. 16, Jul. 2019. DOI: 10.22331/qv-2019-07-21-16.

[5] P. Zanardi, "Virtual quantum subsystems," Physical Review Letters, vol. 87, no. 7, Jul. 2001. DOI: 10.1103/physrevlett.87.077901.

[6] P. Zanardi, D. A. Lidar, and S. Lloyd, "Quantum tensor product structures are observable induced," Physical Review Letters, vol. 92, no. 6, Feb. 2004. DOI: 10.1103/physrevlett.92.060402.

[7] A. M. Gleason, "Measures on the closed subspaces of a Hilbert space," Journal of Mathematics and Mechanics, vol. 6, no. 6, pp. 885-893, 1957.

[8] V. V. Kornyak, "Quantum models based on finite groups," Journal of Physics: Conference Series, vol. 965, p. 012 023, Feb. 2018. DOI: 10.1088/1742-6596/965/1/012023.

[9] V. V. Kornyak, "Modeling quantum behavior in the framework of permutation groups," in EPJ Web of Conferences. EDP Sciences, 2018, vol. 173, p. 01007 . DOI: $10.1051 /$ epj conf /201817301007.

[10] V. V. Kornyak, "Mathematical modeling of finite quantum systems," Lect. Notes Comput. Sci., vol. 7125, pp. 79-93, 2012. arXiv: 1107.5675 [quant-ph] .

[11] T. Banks, "Finite deformations of quantum mechanics," 2020. arXiv: 2001.07662 [hep-th].

[12] M. J. Collins, "On Jordan's theorem for complex linear groups," Journal of Group Theory, vol. 10, no. 4, pp. 411-423, 2007. DOI: 10.1515/JGT . 2007.032.

[13] A. Rényi, "On measures of entropy and information," English, in Proc. 4th Berkeley Symp. Math. Stat. Probab. 1, 1961, pp. 547-561.

[14] M. Van Raamsdonk, "Building up spacetime with quantum entanglement," Gen. Rel. Grav., vol. 42, pp. 2323-2329, 2010. DOI: 10.1142/ S0218271810018529. arXiv: 1005.3035 [hep-th].

[15] J. Maldacena and L. Susskind, "Cool horizons for entangled black holes," Fortschritte der Physik, vol. 61, no. 9, pp. 781-811, Aug. 2013. DOI: 10.1002/prop. 201300020. 
[16] M. A. Nielsen and I. L. Chuang, Quantum computation and quantum information, 10th anniversary edition. USA: Cambridge University Press, 2016.

\section{For citation:}

V. V. Kornyak, Quantum mereology in finite quantum mechanics, Discrete and Continuous Models and Applied Computational Science 29 (4) (2021) 347-360. DOI: $10.22363 / 2658-4670-2021-29-4-347-360$.

\section{Information about the authors:}

Kornyak, Vladimir V. - Doctor of Sciences in Physics and Mathematics, Leading researcher, Laboratory of Information Technologies, Joint Institute for Nuclear Research (e-mail: vkornyak@gmail.com, phone: + 7(909)6791491, ORCID: https://orcid.org/0000-0002-5712-2960) 
УДК 512.542:530.145

PACS 03.65.Fd, 03.65.Ud, 03.65.Aa

DOI: $10.22363 / 2658-4670-2021-29-4-347-360$

\title{
Квантовая мереология в конечной квантовой механике
}

\section{В. В. Корняк}

\author{
Лаборатория информационных технологий \\ Объединённый институт ядерных исследований \\ ул. Жолио-Кюри, д. 6, Дубна, Московская область, 141980, Россия
}

Любое гильбертово пространство составной размерности можно разложить в тензорное произведение меньших гильбертовых пространств. Такая факторизация дает возможность разложить квантовую систему на подсистемы. Мы предлагаем модель, основанную на конечной квантовой механике, для конструктивного изучения таких разложений.

Ключевые слова: квантовая мереология, замкнутая квантовая система, квантовые подсистемы, конечная квантовая механика, квантовая запутанность, энергия 